\title{
Opsoclonus-myoclonus syndrome, a post-infectious neurologic complication of COVID-19: case series and review of literature
}

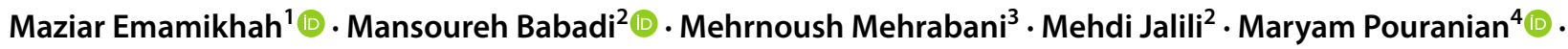 \\ Peyman Daraie $^{5} \cdot$ Fahimeh Mohaghegh $^{1} \cdot$ Sharmin Aghavali $^{1} \cdot$ Maryam Zaribafian $^{6} \cdot$ Mohammad Rohani $^{1,7}$ (i)
}

Received: 23 August 2020 / Revised: 2 November 2020 / Accepted: 27 December 2020

(c) Journal of NeuroVirology, Inc. 2021

\begin{abstract}
Opsoclonus-myoclonus-ataxia syndrome is a heterogeneous constellation of symptoms ranging from full combination of these three neurological findings to varying degrees of isolated individual sign. Since the emergence of coronavirus disease 2019 (COVID-19), neurological symptoms, syndromes, and complications associated with this multi-organ viral infection have been reported and the various aspects of neurological involvement are increasingly uncovered. As a neuro-inflammatory disorder, one would expect to observe opsoclonus-myoclonus syndrome after a prevalent viral infection in a pandemic scale, as it has been the case for many other neuro-inflammatory syndromes. We report seven cases of opsoclonus-myoclonus syndrome presumably parainfectious in nature and discuss their phenomenology, their possible pathophysiological relationship to COVID-19, and diagnostic and treatment strategy in each case. Finally, we review the relevant data in the literature regarding the opsoclonus-myoclonus syndrome and possible similar cases associated with COVID-19 and its diagnostic importance for clinicians in various fields of medicine encountering COVID-19 patients and its complications.
\end{abstract}

Keywords COVID-19 $\cdot$ SARS-CoV-2 $\cdot$ Opsoclonus $\cdot$ Myoclonus $\cdot$ Parainfectious

\section{Introduction}

Myoclonus is defined as brief, sudden, shock-like muscle contraction leading to jerky movements. It is further divided to positive and negative myoclonus based on

\author{
Mohammad Rohani \\ mohammadroohani@gmail.com \\ Maziar Emamikhah \\ maziar.emamikhah@yahoo.com \\ Mansoureh Babadi \\ babadi.mansoureh@gmail.com \\ Mehrnoush Mehrabani \\ Mehrnooshmehrabani@yahoo.com \\ Mehdi Jalili \\ Mehdidjalili@yahoo.com \\ Maryam Pouranian \\ Pouranian.maryam112@gmail.com \\ Peyman Daraie \\ Peyman.da.215@gmail.com \\ Fahimeh Mohaghegh \\ fahmoh2013@gmail.com \\ Sharmin Aghavali \\ shaghavali@gmail.com
}

increased activation or inhibition of muscle contraction. There are several classifications for myoclonus from different aspects; the most commonly used focuses on the generating site of myoclonus. This classifies the myoclonus as cortical, cortical-subcortical, subcortical/

Maryam Zaribafian

Zaribafian_m@yahoo.com

1 Department of Neurology, School of Medicine, Hazrat Rasool-E Akram General Hospital, Iran University of Medical Sciences, Tehran, Iran

2 Ganjavian Hospital, Dezful University of Medical Sciences, Khuzestan Province, Dezful, Iran

3 Fajr Hospital, AJA University of Medical Sciences, Tehran, Iran

4 Amiralmomenin Hospital, Zabol University of Medical Sciences, Zabol, Iran

5 Khatamolanbia Hospital, Shoushtar University of Medical Sciences, Khuzestan Province, Shoushtar, Iran

6 Nikan Hospital, Tehran, Iran

7 Skull Base Research Center, Five Senses Health Institute, Iran University of Medical Sciences, Tehran, Iran 
nonsegmental, segmental, and peripheral. Etiologic classification on the other side encompasses physiologic myoclonus, essential myoclonus, epileptic myoclonus, and symptomatic myoclonus (Caviness 2019). Opsoclonus as an ocular equivalent of myoclonus is irregular, arrhythmic, and chaotic eye movements in all directions of gaze which exacerbates with pursuit (Cameron and Kilbane 2019; Caviness 2019). It is in fact a saccadic disorder of eye movement also known as "saccadomania" and along with ocular flutter is categorized under the umbrella term "saccadic intrusions without intersaccadic intervals". Opsoclonus is more frequently accompanied by ataxia, encephalopathy, and myoclonus; accordingly, the term "opsoclonus-myoclonus syndrome" (OMS) was introduced (Lemos and Eggenberger 2013). Regarding the etiologic classification of myoclonus, OMS is placed under symptomatic myoclonus category and etiologically categorized into idiopathic, paraneoplastic, infectious, and other subgroups (Caviness 2019; Caviness and Brown 2004).

Adult-onset OMS, unlike the similar disorder in children (referred to as Kinsbourne syndrome) is a less well-defined entity, especially in association with infections. The majority of reported cases in adults have been paraneoplastic in nature mostly in association with antineuronal nuclear antibody type 2 [ANNA-2; anti-Ri]. However many idiopathic cases with presumed parainfectious etiology seem to occur and these patients manifest a short duration (4-6 weeks) symptomatic phase and in most cases respond to immunotherapy (Klaas et al. 2012). Symptoms, especially the myoclonus, alleviate with clonazepam or other drugs such as sodium valproate and levetiracetam (Kojovic et al. 2011).

As the new coronavirus infection disease (COVID-19) continues to be the major current health issue around the world, new symptoms regarding neurological aspect of this multi-system disease are being reported. Cerebrovascular disorders (e.g., ischemic stroke, intracerebral hemorrhage, cerebral vasculitis), altered mental status (due to encephalitis, encephalopathy, seizure, etc.), peripheral nervous system involvement (Guillain-Barré syndrome, myositis, etc.), and neuropsychiatric involvement (e.g., depression, personality change, catatonia, mania, and psychosis) have been the major neurological manifestations of COVID-19 frequently reported ( $\mathrm{Lu}$ et al. 2020; Varatharaj et al. 2020). Regarding movement disorder neurological symptoms in COVID-19, the semiology, pathophysiology, and epidemiology of each movement disorder are still naïve. In some studies, there are classifications for staging the COVID-19 disease course. These stages have been proposed as acute respiratory distress syndrome, cytokine storm, acute hypercoagulable state, and autonomic dysfunction (Yamamoto et al. 2020).
Although these may explain some of the neurological aspects of COVID-19, we believe that there are much more aspects to focus on, phenomenologically and pathophysiologically, among them are parainfectious autoimmune syndromes. We have been consulted on seven cases with COVID-19 either in acute infectious phase or early after remission in whom the emergence of abnormal movements resembling OMS were noticeable.

\section{Case report}

We report here seven COVID-19 patients who developed similar symptoms, i.e., myoclonus. These cases were consulted with two of authors (MR and ME) in a referral center for movement disorders. The cases 1,3 , and 4 were reported from centers where two of the authors work and managed them (MB and $\mathrm{MJ}$ ). Cases 2 and 5 were managed in the hospitals where two of the authors are practicing (MM and MZ, respectively). The 6th patient was referred for treatment to ME and MR. The 7th patient was recently managed by our colleague; FM, ME, and MR were consulted on this case. All patients gave their informed consent prior to their inclusion in the study. Patient's characteristics and their disease-related information are summarized in Table 1.

Case 1. A 51-year-old male health-care staff (nurse) presented with sore throat, back pain, anorexia, and mild dyspnea. There was no leukocytosis, lymphopenia, or increment in erythrocyte sedimentation rate (ESR) or C-reactive protein (CRP). The chest CT revealed few peripheral patchy ground-glass opacities (Fig. 1). Brain CT was normal. His nasopharyngeal swab sample RT-PCR test result for COVID-19 was positive. He was treated with non-steroidal anti-inflammatory drugs (piroxicam IM) for myalgia, hydroxychloroquin, and azithromycin. Two weeks after initial symptoms, he noticed tremor-like jerky movements in his hands which progressively increased in severity and became worse with intentional movements. After several days, his legs and voice were involved with tremor and he noticed oscillopsia. His jerky movements diagnosed as generalized stimulus sensitive and action myoclonus, treated with clonazepam $0.5 \mathrm{mg}$ qhs and levetiracetam $500 \mathrm{mg}$ bid. His extra ocular movements were fragmented during pursuit movements intermixed with jerky oscillatory movements of eyes in all directions (opsoclonus) and corrective rapid head movements. There was slightly truncal ataxia (wide-based gait) more prominent during tandem walking (Online Resource 1). Based on these symptoms, parainfectious OMS was presumed and IVIG was started to a total dose of $150 \mathrm{~g}(2 \mathrm{~g} / \mathrm{kg})$. The improvement of abnormal movements was noticeable after 1 week of IVIG initiation and markedly improved 3 weeks after treatment (Online Resource 2-4). Clonazepam and levetiracetam 


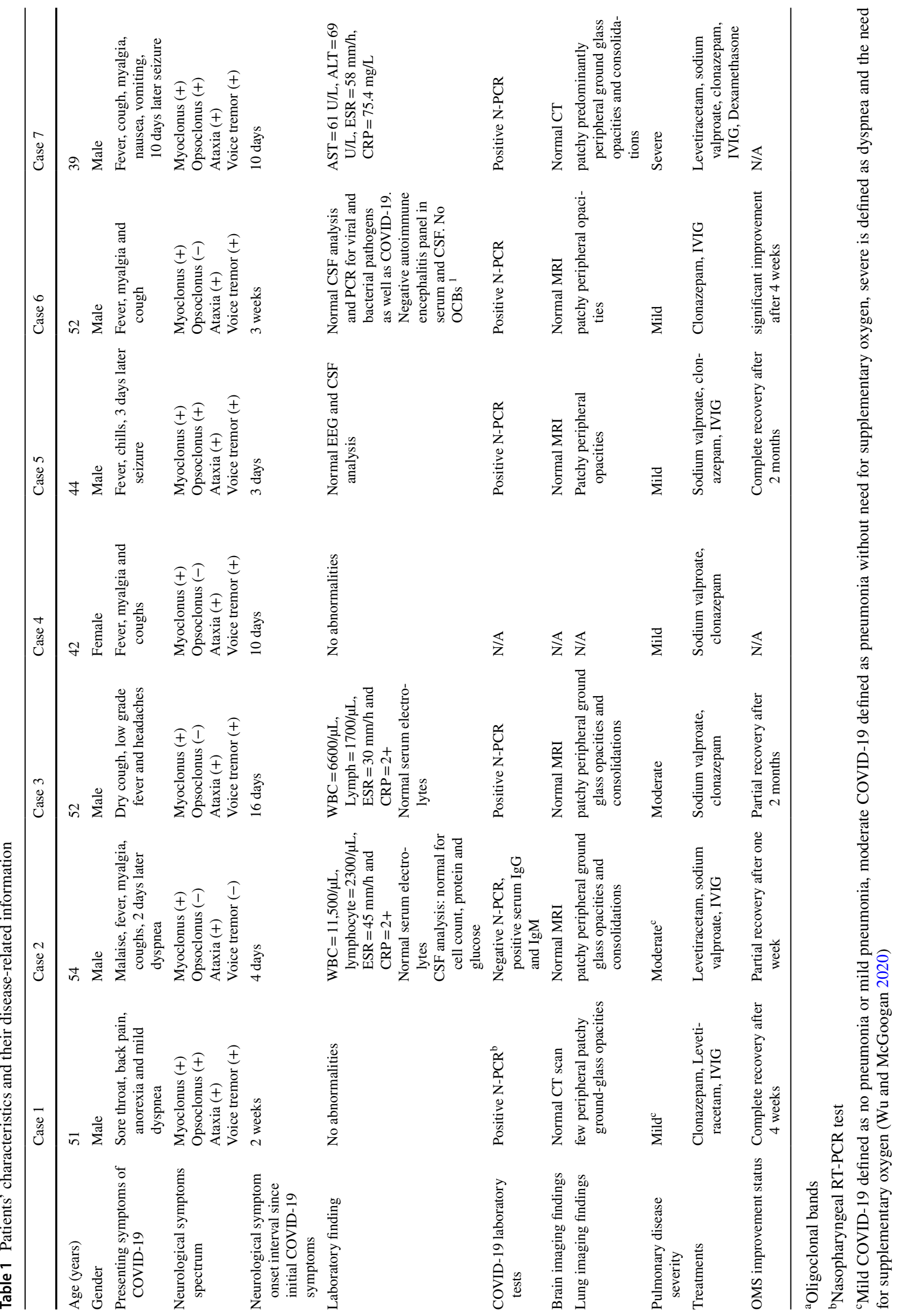




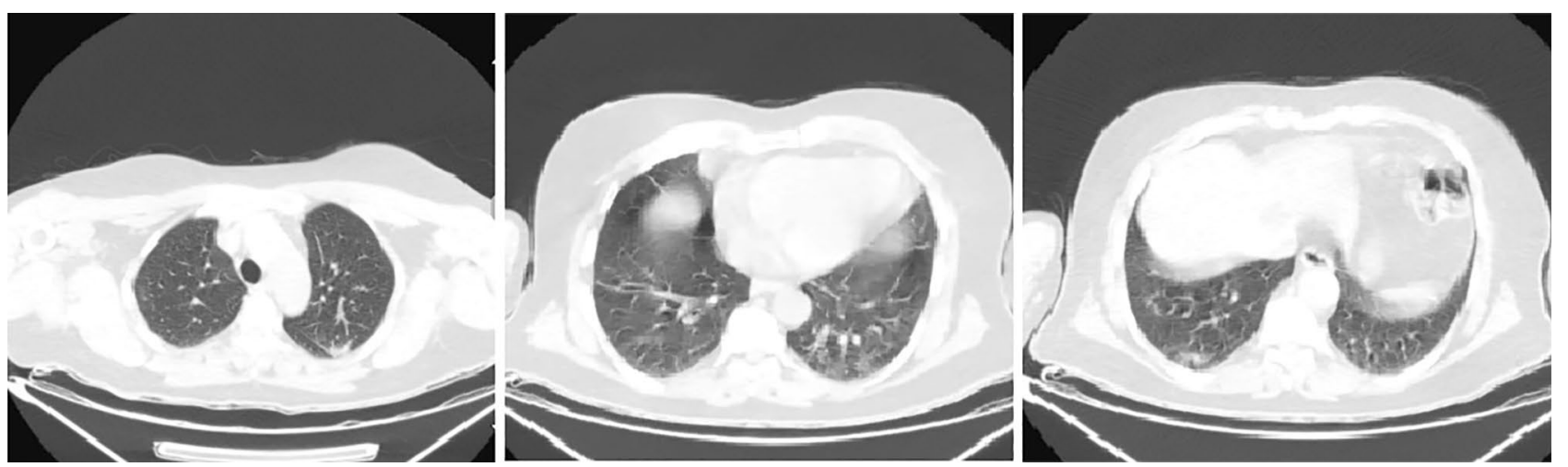

Fig. 1 Chest computed tomography (CT) scan of case 1 shows few peripheral patchy ground-glass opacities in both lungs

4 weeks after treatment were discontinued and there were no abnormal movement's thereafter.

Case 2. A 54-year-old man was admitted to the hospital with dyspnea. His symptoms started with malaise, fever, myalgia, coughs, and 2 days later dyspnea. After nearly 4 days of symptoms' onset, he developed generalized jerky movements. His voice and ocular movements were spared. Examination revealed generalized myoclonic jerks exacerbating with intentional movements and sudden noises (Online Resource 5). Chest CT showed patchy peripheral ground glass opacities and consolidations in both lungs, much more severe than case 1 (Fig. 2). Brain MRI was normal. Blood count revealed leukocytosis $(\mathrm{WBC}=11,500)$ but lymphocyte count was 2300 . ESR was $45 \mathrm{~mm} / \mathrm{h}$ and CRP was highly positive (2+). Serum electrolytes were within normal limits. CSF analysis for cell count, protein, and glucose was normal. Nasopharyngeal and CSF RT-PCR test for COVID-19 were negative but serologic survey for both IgG and IgM in serum were positive. Treatment with levetiracetam (2000 mg/day), sodium valproate (1000 mg/day), and IVIg (100 g total) after 5 days resulted in significant resolution of myoclonic movements and he was discharged with levetiracetam and sodium valproate planned to be tapered within 1 month.

Case 3. This 52-year-old man with history of chronic lung disease came to hospital initially with dry cough, low grade fever, and headaches started 1 week earlier. His laboratory tests revealed $\mathrm{WBC}=6600 / \mu \mathrm{L}$, lymph $=1700 / \mu \mathrm{L}$, $\mathrm{ESR}=30 \mathrm{~mm} / \mathrm{h}$, and $\mathrm{CRP}=2+$ and normal serum electrolytes. Chest CT showed typical COVID-19 involvements (Fig. 3). Nasopharyngeal swab RT-PCR test was positive for COVID19. Treatment for respiratory disease started with oseltamivir, lopinavir/ritonavir, hydroxychloroquine (hospital protocol at that time), and he was discharged after 4 days. Sixteen days after initial symptoms, he developed generalized stimulus (somatosensory and auditory) sensitive myoclonus, involving his voice as well, but not eye movements. His movements became severe and disabling after 3 days making him unable to walk. He was admitted again. Brain MRI was normal. Sodium valproate $1000 \mathrm{mg} / \mathrm{day}$ and clonazepam $1 \mathrm{mg}$ qhs were beneficial, enabling him to walk with help. Chest CT was in favor of superimposed bacterial pneumonia and treatment with IV antibiotics started. He declined immunotherapy and was discharged with oral levofloxacin, sodium valproate, and clonazepam. He did not consent for taking his video but allowed anonymous data consumption for this paper. After 2-month follow-up, his movements were still present but trivial and he was still on medications.

Case 4. A 42-year-old lady visited our clinic, 10 days after initial diagnosis of COVID-19 with initial symptoms of fever, myalgia and coughs, developed jerky movements of hands and feet (more severe on right side), voice tremor, imbalance, and gait disturbance. On neurological examination, she had generalized myoclonus, dysarthria, and mild truncal ataxia similar to case 1 (but less severe). There was no prominent ocular movement involvement. Other neurological examinations were intact. Clonazepam and sodium valproate were started for abnormal movements. Unfortunately, we lost the follow-up of this patient, but we think her symptoms got improved since her respiratory and movement disorders were mild and none of our colleagues in the local area which is a small city have visited her since then.

Case 5. The other similar patient about whom we were consulted was a 44-year-old gentleman, presented with fever, chills, and 3 days later one episode of generalized tonic-clonic seizure. The same day, he developed opsoclonus, generalized stimulus sensitive and action myoclonus and ataxia, which after several days made him completely disabled and unable to sit or walk. His voice was tremulous as well (Online Resource 6-9). Chest CT was in favor of COVID-19 diagnosis (Fig. 4). Brain MRI, EEG, and CSF analysis were normal. Treatment with intravenous ceftriaxone, oral azithromycin, and anti-viral drugs Daclatasvir/ sofosbuvir (Sovodak ${ }^{\circledR}$ ) was instituted. With the presumed diagnosis of OMS, we started sodium valproate, clonazepam, and IVIG. After 1 week, with partial resolution 

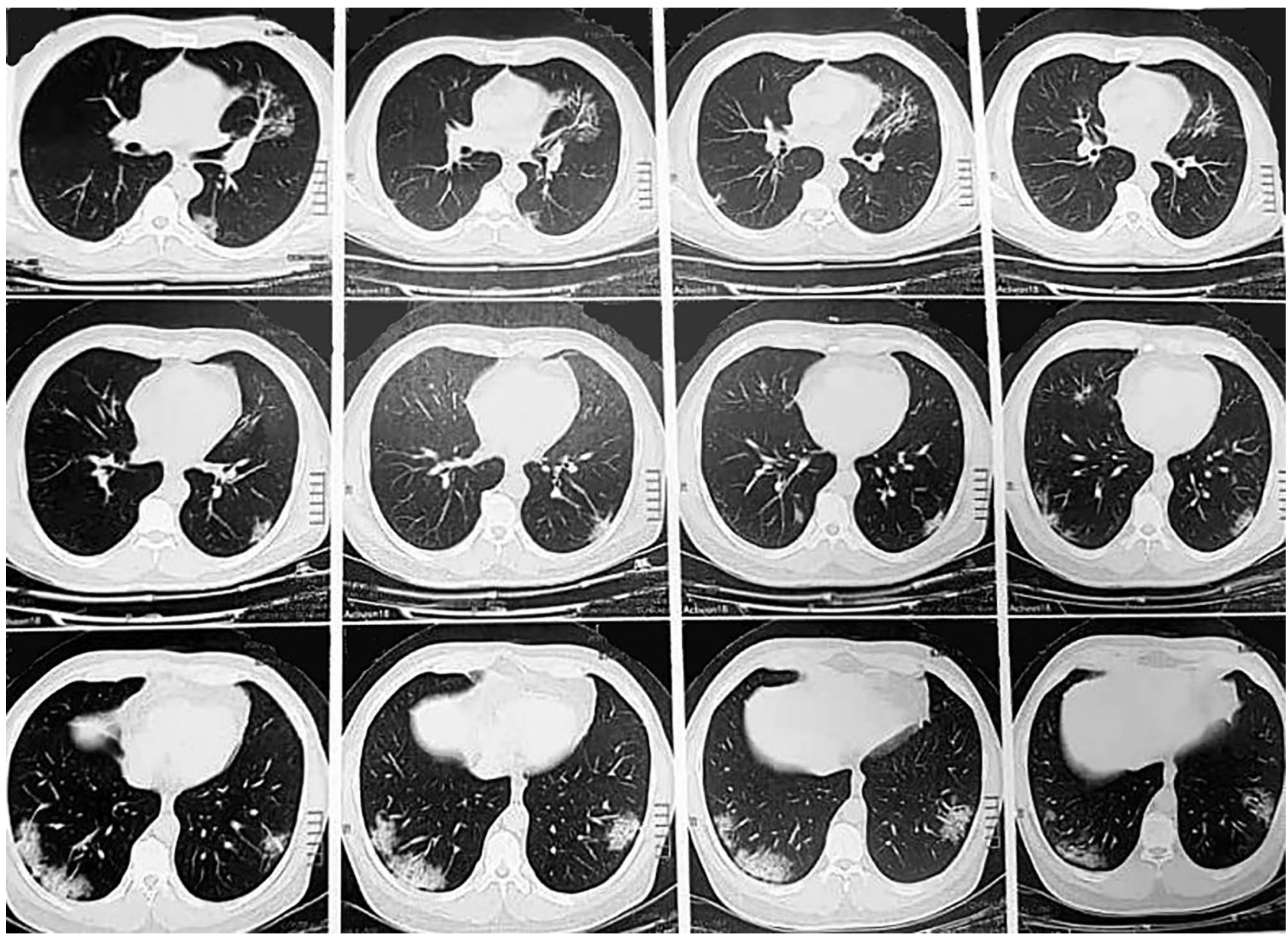

Fig. 2 Chest CT scan of case 2 shows patchy peripheral ground glass opacities and consolidations in both lungs

of symptoms, he was discharged (see Online Resource 6-9). Follow-up at 2 months showed no abnormal movements.

Case 6. Fifty-two-year-old male nearly 1 month after initial symptoms of fever, myalgia, and cough was referred to us for treatment of abnormal movements. Neurological examination revealed generalized myoclonus with intentional component, mild ataxia, and severe shuffling gait. Voice involvement was prominent but there was no opsoclonus (Online Resource 10). Chest CT scan (Fig. 5) was in favor of COVID-19 diagnosis and nasopharyngeal swab RT-PCR test confirmed the diagnosis. CSF protein, glucose, and cell count were normal and PCR for common viral and bacterial pathogens as well as COVID-19 in CSF was negative. Serum and CSF panel for autoimmune encephalitis antibodies were negative. No oligoclonal band was detected. Treatment with clonazepam and IVIG (100 g total) resulted in significant improvement after 4 weeks (Online Resource 11).

Case 7. This very recent patient of us, a 39-year-old man, presented with fever, cough, myalgia, nausea, vomiting, and 10 days later, one episode of generalized tonic-clonic seizure. The same day, he began to suffer from jerky movements. Examination revealed prominent opsoclonus, generalized
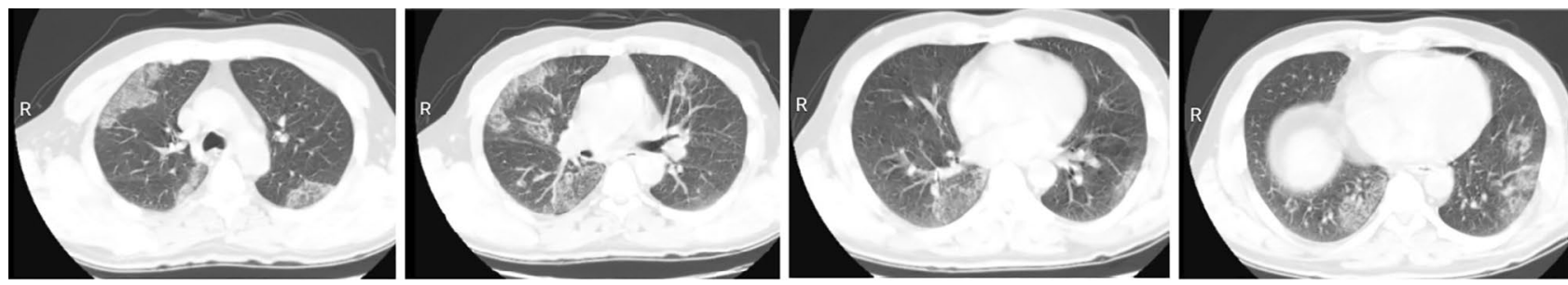

Fig. 3 Chest CT scan of case 3 shows patchy peripheral ground glass opacities and consolidations in both lungs typical for COVID-19 involvements 


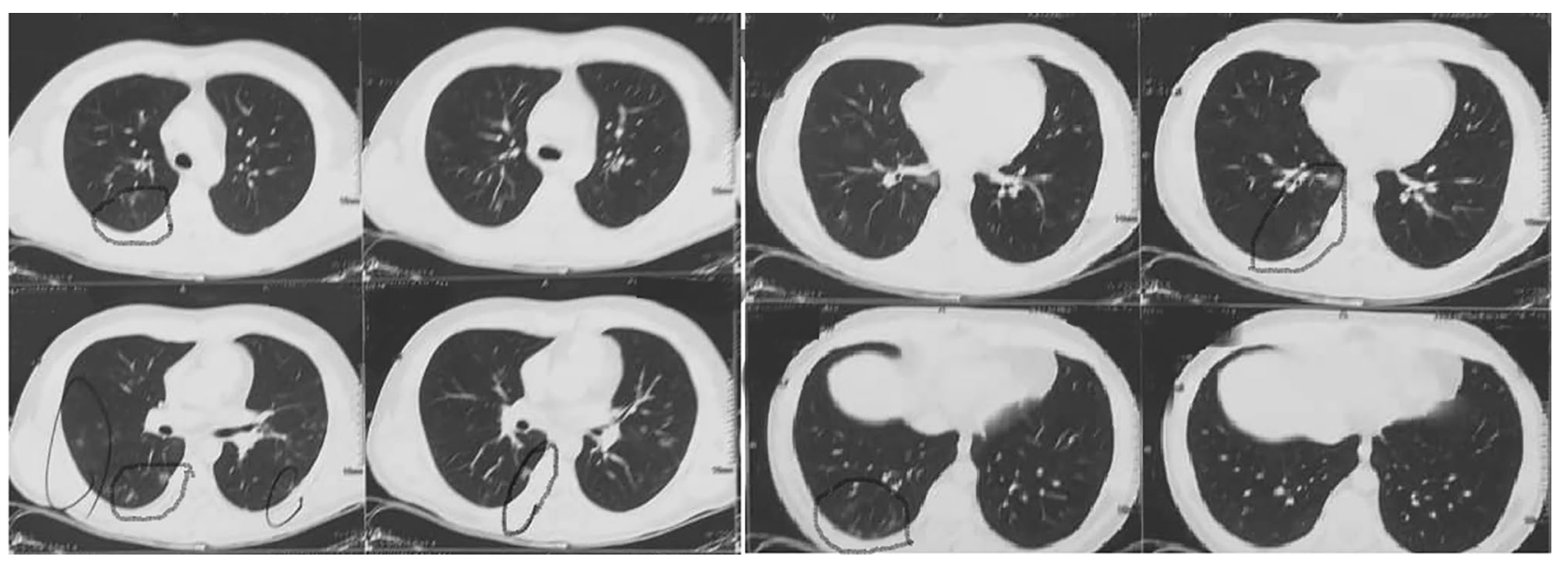

Fig. 4 Chest CT scan of case 5 shows patchy peripheral opacities (circles) in both lungs more severe in right lung in favor of COVID-19 diagnosis

myoclonus with intentional component, and ataxia. His voice was severely involved (Online Resource 12). Chest CT was diagnostic for COVID-19 (Fig. 6). Brain CT was normal. Abnormal laboratory tests included AST $=61 \mathrm{U} / \mathrm{L}, \mathrm{ALT}=69$ $\mathrm{U} / \mathrm{L}, \mathrm{ESR}=58 \mathrm{~mm} / \mathrm{h}, \mathrm{CRP}=75.4 \mathrm{mg} / \mathrm{L}$. He was started on levetiracetam, clonazepam, IVIG, and dexamethasone. After 2 days, sudden increase in serum creatinine $(\mathrm{Cr}=5)$ made us to stop IVIG and change levetiracetam to sodium valproate. He is still under treatment for severe respiratory involvement and acute renal failure. Clonazepam and sodium valproate alleviated myoclonus but improvement in general condition and OMS merits long-term follow-up.

\section{Discussion}

Opsoclonus-myoclonus-ataxia syndrome is a heterogeneous constellation of symptoms ranging from full combination of these three neurological findings to varying degrees of isolated each individual sign. In a review of patients with adult-onset OMS, one third had flu-like symptoms before the emergence of abnormal movements and $40 \%$ revealed either elevated IgG index or oligoclonal bands, none of them being paraneoplastic in follow-up, but results for presumed infections to establish parainfectious source were negative. Eighty percent received immunotherapy (IVIG,

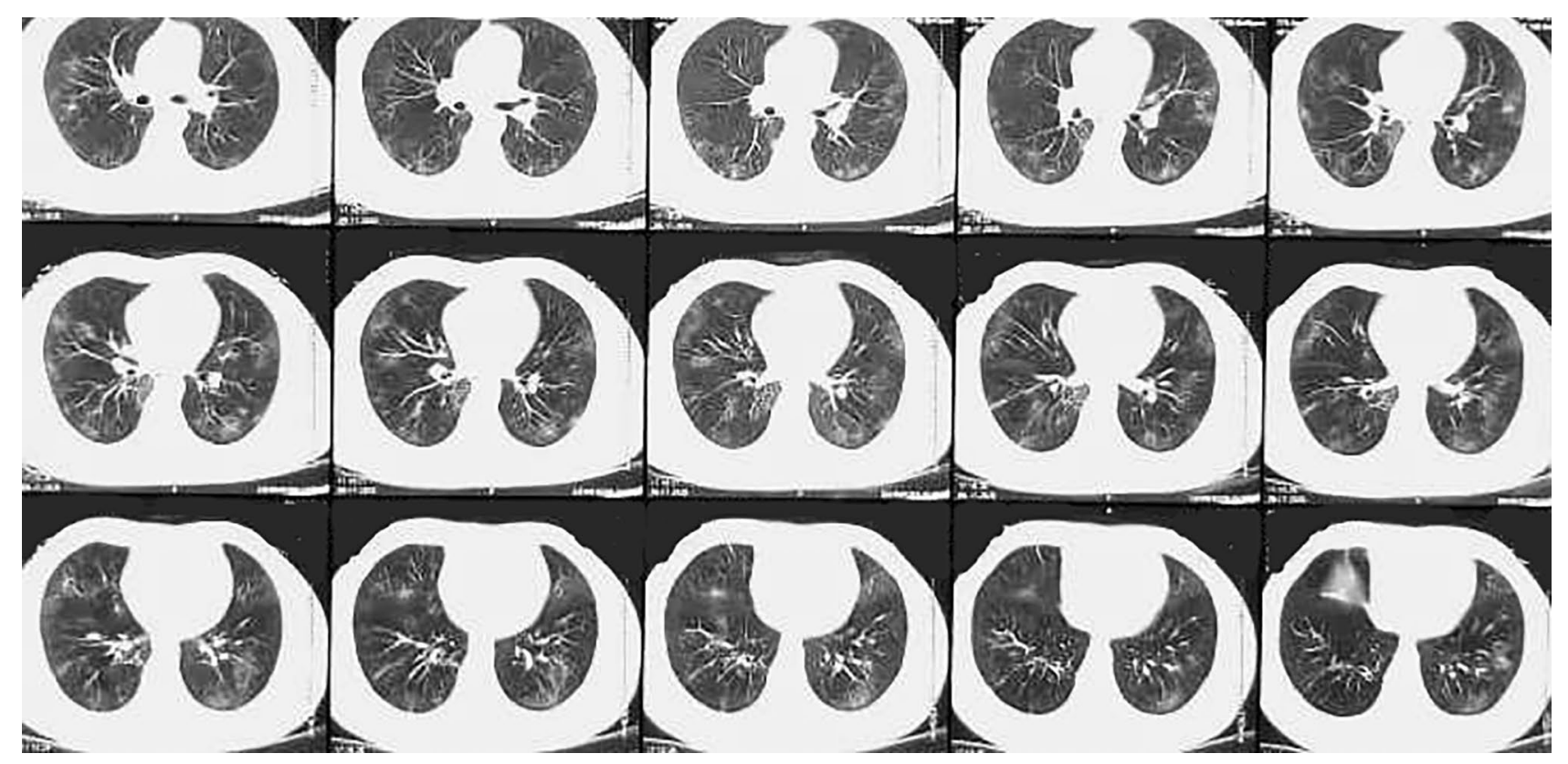

Fig. 5 Chest CT scan of case 6 shows patchy peripheral opacities in both lungs in favor of COVID-19 diagnosis 


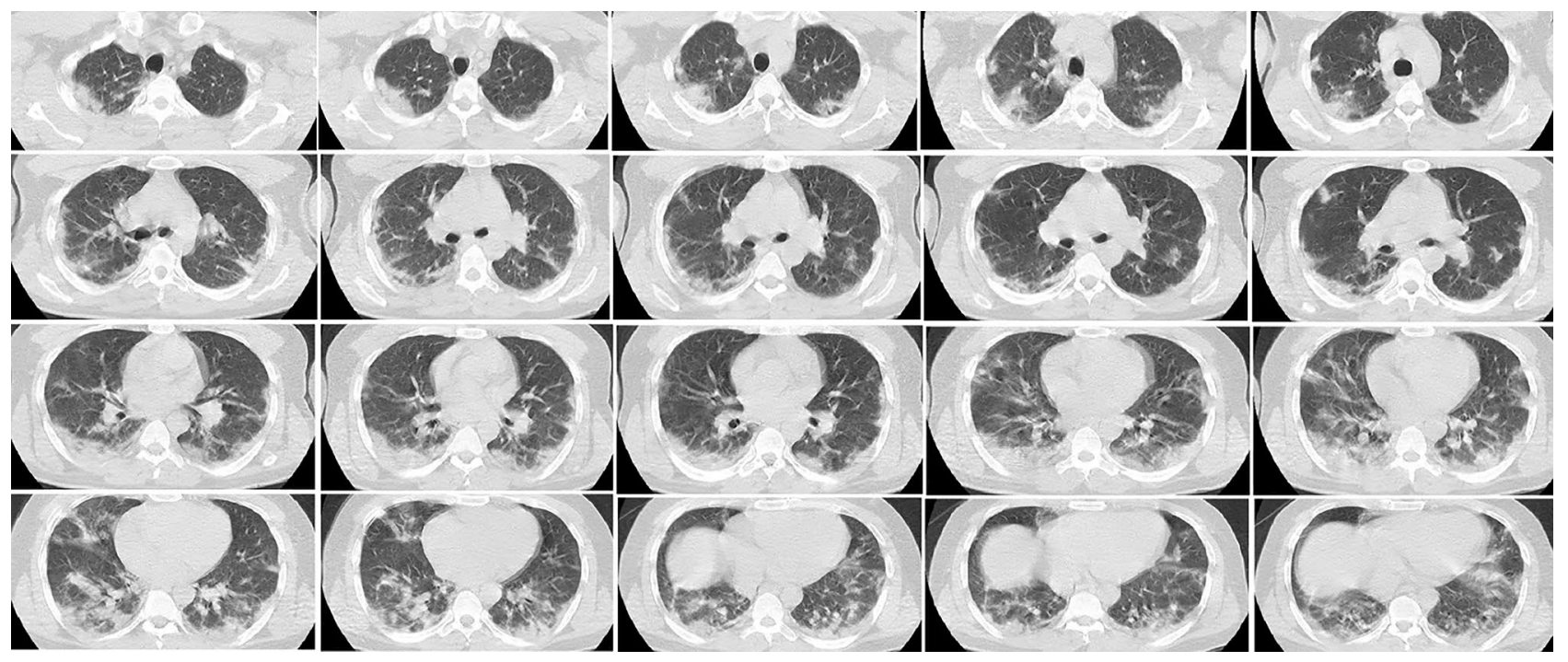

Fig. 6 Chest CT scan of case 7 shows patchy predominantly peripheral ground glass opacities and consolidations in both lungs supporting the COVID-19 diagnosis

corticosteroids, plasma exchange, or a combination of them) and almost all of non-paraneoplastic cases improved. Proven infection was identified in 13\% of OMS patients extracted from review of the literature (Klaas et al. 2012).

Since the emergence of COVID-19, neurological symptoms, syndromes, and complications associated with this multi-organ viral infection have been reported and the various aspects of neurological involvement are increasingly uncovered. There have been a proposed staging for COVID-19 defined as acute respiratory distress syndrome, cytokine storm, acute hypercoagulable state, and autonomic dysfunction (Yamamoto et al. 2020), but this may explain some aspects of neurological COVID-19. Many parainfectious autoimmune complications such as Guillain-Barré syndrome are increasingly reported (Varatharaj et al. 2020) and these immune-mediated complications may occur with varying interval from initial COVID-19 symptoms and may overlap with each of the above mentioned stages. As a neuro-inflammatory disorder in nature, one would expect to observe OMS after a prevalent viral infection in a pandemic scale, as it has been the case for many other neuro-inflammatory syndromes (Paterson et al. 2020).

However, the syndrome as a specific consideration related to COVID-19 has not been discussed fully in the literature. Here, we presented seven similar OMS cases after COVID19 , which we were consulted for, as a movement disorder referral center.

The best similar report regarding this disorder, has been in three patients, xwith generalized myoclonus following the inflammatory phase of COVID-19. None of them had opsoclonus, all three had encephalopathy and responded to immunotherapy indicating a parainfectious immune mechanism (Rábano-Suárez et al. 2020).

Myoclonus as a component of COVID-19-associated delirium has been reported by others and presumed to be a feature of generalized brain dysfunction in encephalopathic patients (Beach et al. 2020). Others also reported COVID-19associated encephalopathy having myoclonus in their clinical picture and assumed brainstem involvement as the pathogenic mechanism (Chaumont et al. 2020). There is a case report of clozapine toxicity in a COVID-19 patient who also had myoclonus (Cranshaw and Harikumar 2020). Also, there is report of a patient in UK-wide surveillance study with OMS but we were not able to access data regarding encephalopathy in this case (Varatharaj et al. 2020). Same authors reported a patient with OMS who had been encephalopathic and assumed to have autoimmune encephalitis with brainstem involvement (Paterson et al. 2020). There is a recent report of a COVID-19 patient who developed generalized action myoclonus 1 month after respiratory disease and the authors presumed it to be post-hypoxic in nature, although there was no solid evidence for this etiology in our view (Ros-Castelló et al. 2020). Another similar patient with generalized action myoclonus and ataxia (with voice involvement but without opsoclonus) occurring 2 weeks after severe respiratory disease, has been reported (Dijkstra et al. 2020). The latter is very similar to our cases except for the fact that it has been following severe COVID-19 respiratory phase but six out of seven cases, we reported here, have been after a mild to moderate respiratory disease according to $\mathrm{Wu}$ and McGoogan definition (Wu and McGoogan 2020). Their patient had been more responsive 
to IVIG than high-dose methylprednisolone pulse therapy,in accordance to our experience.

It seems that the OMS merits more attention as an independent immune parainfectious syndrome, with possible relationship to COVID-19 as the initial trigger infection. The interesting point in our patients was the absence of encephalopathy and variable interval after respiratory disorder, which was very mild in the first patient. This may indicate a syndrome independent of encephalopathy or direct effect of infection. The dramatic effect of immunotherapy on recovery in cases 1,5 , and 6 in comparison with case 3 confirms the immune-mediated mechanism for this myoclonic syndrome. We believe some of the reported patients in the literature may have been suffering from the same immune OMS syndrome, which was overlapped by the respiratory or encephalopathic phase of COVID-19. This alarms the possibility of under-diagnosing an autoimmune encephalitic process, if untreated, it may further complicate the overall outcome of COVID-19 patients. Awareness of the possibility of such disorder and becoming familiar with its features, which are easy to recognize even in critical settings, might help in better diagnosis and correctly choosing the COVID-19 patients that may benefit from immunotherapy.

Supplementary information The online version contains supplementary material available at https://doi.org/10.1007/s13365-020-00941-1.

Acknowledgments We acknowledge our patients for consenting their data and videos used for scientific purposes. We also acknowledge the sacrificing medical staff in front line combat with COVID-19 pandemics.

Authors' contributions Conceptualization: Mohammad Rohani; methodology: Maziar Emamikhah, Mohammad Rohani; formal analysis and investigation: Maziar Emamikhah, Mansoureh Babadi, Mohammad Rohani; writing — original draft preparation: Sharmin Aghavali, Maziar Emamikhah, Fahimeh Mohaghegh; writing-review and editing: Mohammad Rohani; resources (referring the cases and data gathering): Mansoureh Babadi, Mehrnoush Mehrabani, Mehdi Jalili, Maryam Pouranian, Peyman Daraie, and Maryam Zaribafian; supervision: Mohammad Rohani.

Funding This study did not receive any funding.

Data availability The full data of each case is available and may be provided on request.

\section{Compliance with ethical standards}

Conflicts of interest The authors declare that they have no conflict of interest.

Ethics approval This study has been approved by the appropriate ethics committee and has therefore been performed in accordance with the ethical standards laid down in the 1964 Declaration of Helsinki and its later amendments.
Consent to participate All patients signed informed consent for anonymous data publication. Cases $1,2,5,6$, and 7 also signed informed consent for the videos to be published for scientific purposes.

\section{References}

Beach SR, Praschan NC, Hogan C, Dotson S, Merideth F, Kontos N, Fricchione GL, Smith FA (2020) Delirium in COVID-19: a case series and exploration of potential mechanisms for central nervous system involvement. Gen Hosp Psychiatry. https://doi.org/10.1016/j. genhosppsych.2020.05.008

Cameron L, Kilbane C (2019) Opsoclonus Myoclonus Syndrome. In: Advances in Translational Neuroscience of Eye Movement Disorders. Springer, pp 471-486. https://doi.org/10.1007/978-3030-31407-1_23

Caviness JN (2019) Myoclonus CONTINUUM: Lifelong Learning in Neurology 25:1055-1080. https://doi.org/10.1212/CON. 0000000000000750

Caviness JN, Brown P (2004) Myoclonus: current concepts and recent advances. Lancet Neurol 3:598-607. https://doi.org/10.1016/S14744422(04)00880-4

Chaumont H, San-Galli A, Martino F, Couratier C, Joguet G, Carles M, Roze E, Lannuzel A (2020) Mixed central and peripheral nervous system disorders in severe SARS-CoV-2 infection. J Neurol 1-7. https://doi.org/10.1007/s00415-020-09986-y

Cranshaw T, Harikumar T (2020) COVID-19 infection may cause clozapine intoxication: case report and discussion. Schizophr Bull. https://doi.org/10.1093/schbul/sbaa070

Dijkstra F, Van den Bossche T, Willekens B, Cras P, Crosiers D (2020) Myoclonus and cerebellar ataxia following Coronavirus Disease 2019 (COVID-19). Mov Disord Clin Pract. https://doi.org/10.1002/ mdc3.13049

Klaas JP, Ahlskog JE, Pittock SJ, Matsumoto JY, Aksamit AJ, Bartleson J, Kumar R, McEvoy KF, McKeon A (2012) Adult-onset opsoclonus-myoclonus syndrome. Arch Neurol 69:1598-1607. https://doi.org/10.1001/archneurol.2012.1173

Kojovic M, Cordivari C, Bhatia K (2011) Myoclonic disorders: a practical approach for diagnosis and treatment. Therapeutic Adv Neurol Disord 4:47-62. https://doi.org/10.1177/1756285610395653

Lemos J, Eggenberger E (2013) Saccadic intrusions: review and update. Curr Opin Neurol 26:59-66. https://doi.org/10.1097/WCO. $0 \mathrm{~b} 013 \mathrm{e} 32835 \mathrm{c} 5 \mathrm{e} 1 \mathrm{~d}$

Lu L, Xiong W, Liu D, Liu J, Yang D, Li N, Mu J, Guo J, Li W, Wang G (2020) New onset acute symptomatic seizure and risk factors in coronavirus disease 2019: a retrospective multicenter study. Epilepsia. https://doi.org/10.1111/epi.16524

Paterson RW, Brown RL, Benjamin L, Nortley R, Wiethoff S, Bharucha T, Jayaseelan DL, Kumar G, Raftopoulos RE, Zambreanu L (2020) The emerging spectrum of COVID-19 neurology: clinical, radiological and laboratory findings. Brain. https://doi.org/10.1093/ brain/awaa240

Rábano-Suárez P, Bermejo-Guerrero L, Méndez-Guerrero A, ParraSerrano J, Toledo-Alfocea D, Sánchez-Tejerina D, SantosFernández T, Folgueira-López MD, Gutiérrez-Gutiérrez J, AyusoGarcía B (2020) Generalized myoclonus in COVID-19. Neurol. https://doi.org/10.1212/WNL.0000000000009829

Ros-Castelló V, Quereda C, López-Sendón J, Corral I (2020) Posthypoxic myoclonus after COVID-19 infection recovery. Mov Disord Clin Pract. https://doi.org/10.1002/mdc3.13025

Varatharaj A, Thomas N, Ellul MA, Davies NW, Pollak TA, Tenorio EL, Sultan M, Easton A, Breen G, Zandi M (2020) Neurological and neuropsychiatric complications of COVID-19 in 153 patients: a UK-wide surveillance study. Lancet Psychiatry. https://doi. org/10.1016/S2215-0366(20)30287-X 
Wu Z, McGoogan JM (2020) Characteristics of and important lessons from the Coronavirus Disease 2019 (COVID-19) outbreak in China: summary of a report of 72314 cases from the Chinese Center for Disease Control and Prevention. JAMA 323:1239-1242. https://doi. org/10.1001/jama.2020.2648

Yamamoto V, Bolanos JF, Fiallos J, Strand SE, Morris K, Shahrokhinia S, Cushing TR, Hopp L, Tiwari A, Hariri R, Sokolov R, Wheeler C, Kaushik A, Elsayegh A, Eliashiv D, Hedrick R, Jafari B, Johnson JP, Khorsandi M, Gonzalez N, Balakhani G, Lahiri S, Ghavidel K, Amaya M, Kloor H, Hussain N, Huang E, Cormier J, Wesson
Ashford J, Wang JC, Yaghobian S, Khorrami P, Shamloo B, Moon C, Shadi P, Kateb B (2020) COVID-19: review of a 21st century pandemic from etiology to neuro-psychiatric implications. J Alzheimer's Dis 77:459-504. https://doi.org/10.3233/JAD-200831

Publisher's Note Springer Nature remains neutral with regard to jurisdictional claims in published maps and institutional affiliations. 